\title{
Fatores associados ao cometimento de atos infracionais na adolescência
}

\author{
Lais Sette Galinari ${ }^{1}$ \\ Iris Daniela Arruda Vicari ${ }^{2}$ \\ Marina Rezende Bazon ${ }^{3}$ \\ ${ }^{1}$ Universidade de São Paulo, SP, Brasil \\ https://orcid.org/0000-0001-9959-2314 \\ ${ }^{2}$ Universidade de São Paulo, SP, Brasil \\ https://orcid.org/0000-0002-4959-3383 \\ ${ }^{3}$ Universidade de São Paulo, SP, Brasil \\ https://orcid.org/0000-0002-8037-8710
}

\begin{abstract}
Resumo
Existem sólidas evidências internacionais acerca de fatores de risco específicos associados ao desenvolvimento da conduta delituosa na adolescência. No presente estudo buscou-se identificar quais dos fatores se mostrariam significativos no contexto sociocultural brasileiro. Trabalhou-se com dados de uma amostra composta por 529 adolescentes do sexo masculino, com idades entre 16 e 18 anos (260 judicializados; 269 escolares), coletados com um questionário. Foram feitas análises de regressão logística, sendo que no modelo ajustado, as variáveis que se mostraram relevantes foram: a) status socioeconômico; b) paternidade; c) idade; d) trabalho; e) uso de substâncias; f) pares de idade; g) desempenho escolar; h) impulsividade; i) supervisão parental; e i) investimento familiar. Atuando como risco, os fatores mais relevantes foram baixo status socioeconômica e uso frequente de maconha. Atuando como proteção somente investimento familiar.
\end{abstract} Palavras-chave: delinquência juvenil, fatores de risco, fatores de proteção.

\section{Factors associated with juvenile delinquency}

\begin{abstract}
There is strong international evidence on specific risk factors associated with the development of criminal conduct in adolescence. In the present study we tried to identify which of the factors would be significant in the Brazilian sociocultural context. Data were collected from a sample composed of 529 male adolescents, aged between 16 and 18 years (260 judicialized, 269 scholars), collected with a questionnaire. Logistic regression analysis was performed. In the adjusted model, the variables that were relevant were: a) socioeconomic status; b) paternity; c) age; d) work; e) the use of substances; f) the age pairs; g) school performance; h) impulsivity; i) parental supervision; i) family investment. Acting as a risk, the most relevant factors were low socioeconomic status and frequent use of marijuana. Acting as protection only family investment.
\end{abstract}

Keywords: juvenile delinquency; risk factors; protective factors; 


\section{Factores asociados a la delincuencia juvenil}

\section{Resumen}

Existen sólidas evidencias internacionales sobre factores de riesgo específicos asociados al desarrollo de la conducta delictiva en la adolescencia. En el presente estudio se buscó identificar cuáles de los factores se mostrarían significativos en el contexto sociocultural brasileño. Se trabajó con datos de una muestra compuesta por 529 adolescentes del sexo masculino, con edad entre 16 y 18 años (260 judicializados, 269 escolares), recolectados con un cuestionario. Se realizaron análisis de regresión logística. En el modelo ajustado, las variables relevantes fueron: a) status socioeconómico; b) paternidad; c) edad; d) trabajo; e) uso de sustancias; f) los pares; g) rendimiento escolar; h) impulsividad; i) supervisión parental; i) inversión familiar. Como riesgo, los factores más relevantes fueron bajo estatus socioeconómico y uso frecuente de marihuana. Como protección solamente inversión familiar.

Palabras clave: delincuencia juvenil, factores de riesgo, factores protectores.

O número de adolescentes no sistema socioeducativo, em razão de envolvimento em delitos, vem aumentando significantemente. Segundo dados do Conselho Nacional de Justiça, no ano de 2016, havia 189 mil adolescentes cumprindo medidas socioeducativas no País. Isso é aproximadamente o dobro do registrado no ano anterior, quando havia 96 mil adolescentes no sistema socioeducativo (Ministério de Direitos Humanos [MDH], 2016). O envolvimento sério dos adolescentes em delitos, para além dos prejuízos à sociedade, os expõe diretamente a situações de risco que podem materializar-se em agravos para a sua saúde/vida. Assim, trata-se de uma problemática relevante socialmente, em magnitude e em consequências. Por isso, merece investimentos em prevenção.

Estudos na perspectiva da Criminologia Desenvolvimental demonstraram que a conduta delituosa pode se manifestar e desenvolver na adolescência, na medida da exposição do jovem a alguns fatores de risco (Le Blanc, 2006; Moffit, 2018;). Nessa linha, também buscam identificar e sistematizar os fatores associados aos diferentes padrões de conduta delituosa (Le Blanc, 2006). Os mais significativos estão relativamente bem estabelecidos na literatura científica. Muitos estudos, inclusive de meta-análise, destacam fatores dinâmicos pessoais e sociais que aumentam a probabilidade de os adolescentes apresentarem um envolvimento sério em delitos, ou seja, praticarem delitos de forma persistente. Fatores de risco dinâmicos são aqueles que podem ser modificados mediante intervenção, devendo eles, portanto, serem focalizados em programas de prevenção primária e secundária, no contexto de políticas públicas para a juventude, ao passo que os fatores estáticos, aqueles de caráter histórico, embora muito significativos em sua capacidade de predição da conduta futura, não são suscetíveis às intervenções (Andrews \& Bonta, 2006; Onifade, Nyandoro, Davidson II, \& Campbel, 2010).

Considerando essas meta-análises, os fatores de risco dinâmicos mais relevantes são impulsividade, orientação antissocial (Assink, van der Put, Hoeve, Vries, Stams, \& Oort, 2015), o abuso de álcool/drogas (Ortega-Campos, García-García, \& Amenta 2014; Assink et al., 2015), associação a pares infratores (Cottle, Lee \& Heilbrun, 2001; Ortega-Campos, García-García, \& Amenta, 2014; Assink et al., 2015), variáveis familiares negativas (Gendreau, Little, \& Goggin, 1996; Cottle, Lee, \& Heilbrun, 2001) e tempo livre ocioso, remetendo a uma rotina desestruturada (Cottle, Lee, \& Heilbrun, 2001). Quanto aos fatores estáticos, aquele com maior peso, mais frequentemente citado, é histórico de prática de delitos (Gendreau, Little, \& Goggin, 1996; Cottle, Lee, \& Heilbrun, 2001; Assink et al., 2015). Outros fatores estáticos como idade, etnia, gênero, baixo status socioeconômico (Gendreau, Little, \& Goggin, 1996) e histórico de abuso físico e de conduta violenta também são mencionados nas meta-análises (Ortega-Campos, García-García, \& Amenta, 2014). É interessante destacar que na meta-análise realizada por Gendreau, Little e Goggin (1996), os fatores dinâmicos tiveram

o mesmo desempenho que os estáticos na predição da conduta delituosa, apresentando pesos equivalentes. 
Em suma, tem-se que os fatores de risco pessoais dinâmicos com mais robusta associação com a conduta delituosa persistente em adolescentes são impulsividade, ou baixo autocontrole, agressividade, egocentrismo, uso de substâncias psicoativas, além de atitudes e orientação antissociais. Quanto aos fatores sociais, tem-se associação a pares antissociais/infratores, variáveis familiares negativas, como baixa qualidade dos relacionamentos e práticas parentais inadequadas, variáveis escolares também negativas, como baixo desempenho, e aspectos de rotina como muito tempo ocioso e frequentação de locais caracterizados por atividade divergente (Andrews \& Bonta, 2006; Le Blanc, 2006; Maruschi, Estevão, $\&$ Bazon, 2013). Sabe-se que o impacto dos fatores de risco é moderado pelos fatores de proteção, quando presentes. Esses, contudo, segundo Dickens e O'Shea (2017), são pouco avaliados nos estudos. De todo modo, na meta-análise realizada por esses autores, os mais relevantes para a delinquência juvenil foram envolvimento em atividades pró-sociais, apoio social, forte apego a pessoas com orientação pró-social e vínculo com instituições sociais, atitude positiva com relação a figuras de autoridade e normas/controle, comprometimento com a escola e traços de personalidade resilientes.

É certo que as ações de prevenção, nessa perspectiva teórica, se baseiam na premissa de que é preciso atenuar ou eliminar fatores de risco e/ou incrementar a resistência a esses por meio da ativação/fortalecimento de fatores de proteção, visando neutralizar/atenuar o impacto das variáveis negativas (Eisenstein \& Souza, 1993). Desse modo, considerando as evidências de que existem fatores que podem aumentar ou diminuir a probabilidade de um adolescente envolver-se em delitos, o objetivo desse estudo foi o de identificar quais fatores se mostrariam significativamente associados a uma prática persistente de delitos, na adolescência, no contexto sociocultural brasileiro.

\section{Método}

Adotou-se, na investigação, uma abordagem quantitativa, de corte transversal, do tipo "caso-controle". Visando constituir um grupo de interesse ("caso") e um de comparação ("controle"), trabalhou-se com dados coletados junto a adolescentes do sexo masculino recrutados em dois contextos diferentes: programas de execução de medidas socioeducativas e escolas públicas. O grupo de interesse (GI) foi composto por 260 adolescentes que estavam em cumprimento de alguma medida socioeducativa ( $25 \%$ estavam em liberdade assistida; $32 \%$ em unidade de execução de medida de internação; $43 \%$ em unidade de internação provisória aguardando a decisão judicial) e o grupo de comparação (GC), por 269 adolescentes estudantes de escolas públicas. Tentou-se o pareamento dos grupos em algumas variáveis sociodemográficas. Nesse sentido, trabalhou-se apenas com adolescentes do sexo masculino por uma questão de conveniência, considerando a maior prevalência desses no sistema socioeducativo e, portanto, a maior acessibilidade a eles, mas também para diminuir efeitos de variáveis intervenientes. Em delinquência juvenil, o sexo funciona como indicador de variáveis biopsicossociais relevantes, que modulam os efeitos dos fatores de risco e de proteção, o que impõe uma abordagem diferencial do tema em função desse (Mullis, Cornille, Mullis, \& Huber, 2004).

Em algumas variáveis sociodemográficas, contudo, verificaram-se diferenças significativas entre eles. A idade média em GI ( $\mathrm{M}=17,02 ; \mathrm{DP}=0,78 ; \mathrm{Med}=17)$ se mostrou significativamente diferente em $\mathrm{GC}(\mathrm{M}=16,71 ; \mathrm{DP}=0,72$; $\mathrm{Med}=17)(\mathrm{U}=61.876,5 ; \mathrm{p}=0,00)$. Houve também diferença na distribuição dos grupos em termos de classe socioeconômica, tendo por base os dados segundo o critério Brasil $\left(x^{2}=83,42 ; p=0,00\right)$. A distribuição pelas classes em GI foi: $12 \%$ B1-B2, $68 \%$ C1-C2 e 20\% D-E; ao passo que em GC foi: 34\% B1-B2, $60 \%$ C1-C2 e 5\% D-E. Os grupos igualmente se diferenciam em relação à experiência de trabalho ( $41 \%$ em GI e $20 \%$ em GC; $x^{2}=10,25 ; p=0,001$ ), ao fato de já ter filho $\left(14 \%\right.$ em GI e $1 \%$ em GC; $\left.x^{2}=10,25 ; p=0,00\right)$ e 
ao de ter/frequentar uma religião (38\% em GI e $47 \%$ em $\left.\mathrm{GC} ; \mathrm{x}^{2}=4,85 ; \mathrm{p}=0,028\right)$. Considerando que tais variáveis poderiam enviesar os resultados, até porque idade e classe socioeconômica, especificamente, seriam fatores de risco estático, os seus dados foram controlados nas análises subsequentes, ao criar um modelo de regressão logística ajustado com essas covariáveis (para mais informações, ver Hosmer \& Lemeshow, 2000).

Quanto à variável dependente, buscou-se efetivamente trabalhar com a variável de interesse, no caso, "o envolvimento sério em prática de delitos", ultrapassando o dado relativo ao fato de os adolescentes serem ou não judicializados, visto que esse poderia implicar em um viés relacionado à atuação das agências de controle, que por vezes incide mais sobre determinados segmentos da sociedade (French, 2017). Assim, em uma primeira análise, os grupos (GI e GC) foram caracterizados em termos de padrão de conduta delituosa, a partir de dados de "delinquência autorrevelada/autodeclarada", visando verificar diferença significativa entre eles, em termos de "envolvimento em prática de delitos", e não somente no tocante ao fato de um reunir adolescentes judicializados e o outro não.

A "delinquência autorrevelada" ou "autodeclarada" remete a uma estratégia de investigação por meio da qual se levanta informação, de autorrelato, sobre o envolvimento de um indivíduo em prática de delitos, independentemente de ele se ter feito apreender/ processar por eles (Le Blanc \& Fréchette, 1989). Do mesmo modo que os indivíduos podem ser vítimas de crimes e jamais denunciarem/registrarem tal evento, oficialmente, podem também se envolver em delitos e nunca serem apreendidos/processados ou serem apreendidos/processados por parte deles apenas. No plano da sociedade, os dados assim coletados representam melhor a realidade do fenômeno, em comparação àqueles levantados nos registros das agências de controle (BOs ou processos judiciais, por exemplo), os quais compõem a dita "delinquência legal", uma vez que os delitos oficiais representam uma parcela, muitas vezes, pequena dos delitos efetivamente praticados (Le Blanc $\&$ Fréchette, 1989). No plano individual, a estratégia da delinquência autorrevelada, apoiada em entrevistas estruturadas ou questionários bem elaborados e bem conduzidos, permite a obtenção de informações detalhadas sobre a conduta delituosa, as quais raramente se fazem presentes nos registros oficiais dos delitos. Essas ajudam a descrever a trajetória da conduta delituosa, de um indivíduo, focalizando idades, frequência e tipos de delitos praticados, de modo a se poder aferir seu padrão.

Por essas razões, as investigações no campo da Criminologia Desenvolvimental privilegiam a delinquência autorrevelada/autodeclarada (Le Blanc \& Fréchette, 1989). Assim, no presente estudo adotou-se esta estratégia; dados sobre uma eventual implicação dos adolescentes nas categorias de delitos mencionadas foram levantados, independentemente de terem sido ou não apreendidos pela polícia. Para a interpretação desses dados, estabeleceu-se, com base em apontamentos da literatura (Thornberry \& Krohn, 2001; Piquero, Farrington, Nagin, \& Moffit, 2010), que "o envolvimento sério em prática de delitos" se caracterizaria por um padrão de conduta frequente, diversificada e por vezes violeta (envolvendo diretamente vítimas).

\section{Instrumentos}

O instrumento empregado na investigação é um questionário. Trata-se do Questionário de Comportamentos Juvenis (QCJ), uma versão adaptada para uso no contexto brasileiro a partir do original, desenvolvido para o projeto Observatório de Delinquência Juvenil, resultado de uma parceria entre a Escola de Criminologia da Universidade do Porto e o governo português para estabelecer dados sobre a relação entre padrões específicos de comportamentos antissociais e características individuais, relacionais e sociais dos jovens. A estrutura do instrumento se assemelha a do questionário utilizado no International Self-Report Delinquency Study (ISRD, Breen, 2010), que explora informações 
sobre seis grandes dimensões: (1) o adolescente: idade, escolaridade, religião, temperamento, atitudes e valores; (2) a família: status socioeconômico, número de pessoas e vínculo, investimento e supervisão parental; (3) rotina e amigos: o que faz e onde vai quando tem tempo livre e o tipo de amigos (se são pró ou antissociais); (4) escola: desempenho acadêmico, estresse escolar e valores em relação aos estudos; (5) bairro: características do bairro/vizinhança e dos vizinhos; e (6) comportamentos antissociais: condutas divergentes e condutas delituosas. Ademais, nele se incluíram as questões do Critério Brasil (Associação Brasileira de Empresas de Pesquisa [ABEP], 2017) para a avaliação da classe econômica dos participantes.

O QCJ é composto por 59 questões com 264 subitens. As respostas às questões nos cinco primeiros domínios são dadas em escalas que geram scores, os quais foram avaliados como variáveis binárias. Com relação aos comportamentos antissociais, solicitam-se informações descritivas sobre as condutas divergentes (ex.: uso de álcool ou outras drogas) e as condutas delituosas (na versão utilizada focalizam-se as seguintes categorias de delitos: Dano, Furto, Roubo, Receptação, Porte ilegal de arma de fogo, Lesão corporal, Rixa, Maus-tratos a Animais e Tráfico de drogas). Os adolescentes são indagados se já teriam apresentado a referida conduta alguma vez (no curso de sua vida). Em caso de resposta afirmativa, são questionados com relação à frequência. Especificamente com relação aos delitos, demanda-se também pelo número nos últimos 12 meses. Essas informações fornecem a prevalência e a frequência da conduta. As questões do Critério Brasil (ABEP, 2017) são corrigidas segundo normas técnicas próprias.

O QCJ foi inicialmente adequado ao contexto brasileiro por Komatsu (2014). Subsequentemente, o QCJ foi aplicado em outros estudos junto a diferentes amostras (Komatsu \& Bazon, 2017b). As qualidades psicométricas do QCJ foram descritas com base em uma amostra de 836 estudantes do sexo masculino (11 e 18 anos) de três cidades da região Sudeste do Brasil. Os alfas de Cronbach variaram de 0,23 a 0,89 e as correlações intradomínio foram significativas em $81 \%$ dos casos, com coeficientes variando de 0,10 a 0,54 . As correlações entre as escalas e os Comportamentos Externalizantes foram significativas em $62 \%$ dos casos, variando de 0,10 a 0,43 . O QCJ mostrou-se válido para rastrear problemas sociais, relacionais e comportamentais na população adolescente.

\section{Procedimento de coleta dos dados}

Antes de dar início ao recrutamento dos adolescentes, o projeto da pesquisa foi avaliado e aprovado no Comitê de Ética em Pesquisa da Faculdade de Filosofia, Ciências e Letras de Ribeirão Preto - FFCLRP-USP (CAAE: 77903617.5.0000.5407). Assim, em vista aos procedimentos éticos, o contato com os adolescentes judicializados foi autorizado pelas instituições onde foram recrutados. Em relação àqueles que estavam em unidades de internação, o contato também foi avalizado pela autoridade judicial. Os adolescentes foram então convidados a colaborar com a pesquisa e aos que aceitavam aplicou-se o instrumento, individualmente em uma sala reservada, no âmbito dos programas de execução das medidas, negociados os horários e as condições de permanência da pesquisadora no local. Considerando o fato de muitos adolescentes em conflito com a lei não serem proficientes em leitura, a aplicação do QCJ foi feita na forma de uma entrevista estruturada (formato oral), no intuito de padronizar os procedimentos de coleta e, assim, melhorar a confiabilidade dos dados. A entrevista estruturada teve duração que variou entre uma hora e uma hora e meia. A coleta de dados nesse contexto foi realizada entre fevereiro de 2018 a maio de 2019.

O contato com os adolescentes não judicializados foi efetuado nas escolas onde estavam inseridos. Para tal, obteve-se autorização dos diretores dos estabelecimentos educacionais. Depois disso, pôde-se contatar os adolescentes para convidá-los e para entregar-lhes Termos de Consentimento a serem levados aos pais/responsáveis. Em dias subsequentes, aplicava-se o instrumento, em pe- 
quenos grupos, em salas de aula reservadas, junto àqueles que traziam o Termo assinado. Durante a aplicação o pesquisador ficava à disposição para eventuais dúvidas e dificuldades quanto ao conteúdo do questionário. A aplicação em grupo teve duração que variou de uma hora e meia a duas horas. A coleta de dados nesse contexto foi realizada entre fevereiro e novembro de 2015.

\section{Procedimento de análise dos dados}

Primeiramente, com o objetivo de comparar GI e GC nos valores médios das variáveis relacionadas ao padrão de conduta delituosa - frequência e diversidade de delitos autorrevelados - foi feito o teste de distribuição normal Kolmogorov-Smirnov (K-S). Os resultados indicaram não haver evidências de que os dados seguiam uma distribuição normal para nenhuma delas, desse modo, optou-se por comparar GI e GC por meio do teste não paramétrico de Mann-Whitney. Foi adotado o nível de significância de 0,05. Em seguida, foram feitas análises de regressão logística reportando odds ratio para avaliar se as variáveis medidas aumentavam ou diminuíam a chance de o desfecho estudado - "o envolvimento sério em prática de delitos" - ocorrer. As variáveis avaliadas no modelo foram as contempladas pelo QCJ. A análise foi feita gerando um odds ratio bruto e outro ponderado para cada variável independente. $\mathrm{O}$ odds ratio bruto indica a razão de chance de uma variável isoladamente para determinado desfecho, enquanto o odds ratio ponderado indica a razão de chance controlando possíveis efeitos de confusão entre todas as variáveis do modelo (Hosmer \& Lemeshow, 2000). Por conta disso, as variáveis idade, nível socioeconômico, ter trabalhado, filhos e religião foram incluídas como covariáveis no modelo ajustado uma vez que poderiam ser variáveis confundidoras, ou seja, a influência da idade, do nível econômico, do fato de ter trabalhado, ter filhos e ter religião podia afetar o efeito das variáveis de interesse para cima ou para baixo, uma vez que não houve controle delas na composição das amostras.
Segundo Hosmer e Lemeshow (2000), um modelo de regressão logística com covariáveis é capaz de neutralizar o efeito das variáveis confundidoras, desde que elas sejam acrescentadas no modelo ajustado.

Para ser possível trabalhar apenas com variáveis binárias, além daquelas avaliadas pelo QCJ, a classe socioeconômica e a idade foram transformadas em variáveis indicadoras. Como nenhum dos participantes era pertencente a classe $\mathrm{A}$, foram definidas 3 categorias (B1-B2, C1-C2 e D-E). As variáveis filhos, trabalho e religião também foram avaliadas de forma binária. Foi adotado um intervalo de confiança para o odds ratio de $95 \%$ e um nível de significância de 0,05 . A análise de dados foi realizada no software STATA versão 12.

\section{Resultados}

Com relação ao padrão de conduta delituosa, verificou-se que GI se mostrou significativamente diferente do de GC, na direção esperada. Os adolescentes em GI efetivamente apresentariam um "envolvimento sério em prática de delitos", sendo a conduta delituosa nesse grupo mais frequente, mais diversificada e mais violenta (envolvendo mais os delitos considerados violentos, em meio aos investigados: Roubo, Lesão Corporal e Rixa). GI teve uma maior diversidade de delitos realizados (GI: méd 5,8 dp 3,4/ GC: méd 1,29 dp 1,8 - U=61.876,5 p=0,000), uma maior frequência média de delitos realizados no último ano (GI: méd 109,17 dp 152,61/ GC: méd 14,12 dp 101,27 $-\mathrm{U}=57.961,5 \mathrm{p}=0,000$ ), uma maior diversidade de delitos violentos (GI: méd 1,84 dp 1,33/ GC: méd 0,33 dp 0,70$\mathrm{U}=58.266,5 \mathrm{p}=0,000$ ) e uma maior frequência média de delitos violentos realizados no último ano (GI: méd 7,48 dp 32,92/ GC: méd 0,59 dp 3,27 - U=49.891,0 p=0,001).

Quantos às variáveis independentes, a Tabela 1 apresenta os resultados das análises de regressão logística reportando o odds ratio para cada uma, individualmente (OR Bruto), e considerando todas as covariáveis (OR Ponderado). Levando em conta as covariáveis, controlando o efeito confundidor de todas as variáveis 
avaliadas entre si, no modelo ajustado as variáveis que aumentaram a chance de o adolescente pertencer a GI, atuando como fator de risco para "o envolvimento sério em prática de delitos" foram: a) Classe $\mathrm{C} 1-\mathrm{C} 2$; b) Classe D-E; c) Idade - 18 anos; d) Trabalho; e) Filhos; f) Impulsividade; g) Uso Semanal de Maconha; h) Supervisão Parental; i) Baixo desempenho escolar; e j) Pares infratores. No modelo ajustado, apenas uma variável atuou no sentido de diminuir as chances de o adolescente pertencer a GI e pode, por seu turno, ser considerada um fator de proteção: Investimento Familiar, essas estão destacadas em negrito.
Na Tabela 1 foi organizada de modo a apresentar, em ordem decrescente, as variáveis que no modelo ajustado tiveram um OR significativo e os seus respectivos valores, discriminando quais delas podem ser consideradas fatores estáticos e dinâmicos. A variável classe socioeconômica é normalmente considerada fator estático, por remeter à classe socioeconômica da família de origem, embora seja passível de mudança a longo prazo, em geral de uma geração a outra. A variável Trabalho também foi alocada no grupo dos fatores estáticos, por remeter ao histórico de vida.

\section{Resultados das análises de regressão logística binária}

Tabela 1. Resultados análises de regressão logística binária

\begin{tabular}{|c|c|c|c|c|c|c|}
\hline \multicolumn{2}{|c|}{ Fator } & Variáveis Independentes & OR Bruto (IC95\%) & $\mathbf{P}$ & OR Ajustado (IC95\%) & $\mathbf{P}$ \\
\hline \multirow{16}{*}{ Risco } & \multirow{6}{*}{ Estático } & Classe D-E & $4.23(2.31 ; 7.73)$ & 0.00 & $22.58(6.12 ; 83.32)$ & 0.00 \\
\hline & & Filhos & $9.03(3.49 ; 23.35)$ & 0.00 & $4.41(1.31 ; 14.82)$ & 0.01 \\
\hline & & Classe C1-C2 & $1.45(1.01 ; 2.08)$ & 0.03 & $3.82(1.65 ; 8.83)$ & 0.00 \\
\hline & & Idade -18 anos & $2.27(1.48 ; 3.47)$ & 0.00 & $2.89(1.26 ; 6.65)$ & 0.01 \\
\hline & & Trabalho & $1.79(1.25 ; 2.58)$ & 0.00 & $2.26(1.77 ; 4.36)$ & 0.01 \\
\hline & & Idade -17 anos & $1.21(0.86 ; 1.72)$ & 0.25 & $1.92(0.95 ; 3.87)$ & 0.06 \\
\hline & \multirow{10}{*}{ Dinâmico } & Uso semanal maconha & $8.69(5.45 ; 13.85)$ & 0.00 & $24.07(9.94 ; 58.31)$ & 0.00 \\
\hline & & Pares Infratores & $12.77(8.31 ; 19.63)$ & 0.00 & $13.89(6.92 ; 27.88)$ & 0.00 \\
\hline & & $\begin{array}{c}\text { Baixo desempenho } \\
\text { escolar }\end{array}$ & $7.84(4.51 ; 13.65)$ & 0.00 & $7.57(2.90 ; 19.77)$ & 0.00 \\
\hline & & Impulsividade & $2.59(1.80 ; 3.74)$ & 0.00 & $3.50(1.76 ; 6.95)$ & 0.00 \\
\hline & & Supervisão Parental & $0.88(0.60 ; 1.29)$ & 0.53 & $2.96(1.38 ; 6.39)$ & 0.00 \\
\hline & & Oposição autoridade & $1.44(1.02 ; 2.05)$ & 0.03 & $0.71(0.36 ; 1.42)$ & 0.34 \\
\hline & & Atitude violenta & $2.03(1.36 ; 3.01)$ & 0.00 & $0.55(0.24 ; 1.26)$ & 0.16 \\
\hline & & Valores antissociais & $1.25(0.75 ; 2.09)$ & 0.38 & $0.57(0.20 ; 1.60)$ & 0.28 \\
\hline & & $\begin{array}{c}\text { Problemas de } \\
\text { comportamento escola }\end{array}$ & $1.85(1.23 ; 2.78)$ & 0.00 & $1.00(0.48 ; 2.06)$ & 0.99 \\
\hline & & Rotina desestruturada & $2.01(1.27 ; 3.17)$ & 0.00 & $0.51(0.19 ; 1.34)$ & 0.17 \\
\hline
\end{tabular}




\begin{tabular}{|c|c|c|c|c|c|c|}
\hline \multirow{5}{*}{ Proteção } & \multirow{5}{*}{ Dinâmico } & Investimento familiar & $0.50(0.35 ; 0.71)$ & 0.00 & $0.51(0.27 ; 0.95)$ & 0.03 \\
\hline & & Apego parental & $0.36(0.24 ; 0.52)$ & 0.00 & $0.63(0.29 ; 1.33)$ & 0.23 \\
\hline & & Vínculo escolar & $0.69(0.40 ; 1.18)$ & 0.18 & $1.38(0.52 ; 3.62)$ & 0.50 \\
\hline & & Religião & $0.67(0.47 ; 0.95)$ & 0.02 & $0.60(0.32 ; 1.14)$ & 0.12 \\
\hline & & Apoio Social & $0.75(0.41 ; 1.36)$ & 0.34 & $0.645(0.20 ; 1.99)$ & 0.44 \\
\hline
\end{tabular}

As variáveis Oposição a Figuras de Autoridade, Valores Antissociais, Atitude Violenta, Problemas de Comportamento na Escola e Rotina Desestruturada apesar de terem um OR bruto que indicava um aumento na chance de pertencer a GI, no modelo ajustado não apresentaram significância estatística, o que significa que o OR alto era devido ao efeito das variáveis confundidoras e não ao efeito da variável em si. Paralelamente, algumas variáveis cujo OR bruto indicava diminuírem a chance de o adolescente pertencer a GI, como Religião e Apego Parental, no modelo ajustado também não apresentaram evidências de significância estatística. Desse modo, das 21 variáveis inseridas no modelo, 10 tiveram o efeito de aumentar as chances de "envolvimento sério em prática de delitos", atuando como fator de risco, e uma de diminuir, atuando como fator de proteção.

\section{Discussão}

No estudo apresentado, o objetivo era identificar fatores significativamente associados ao envolvimento sério de adolescentes na prática de delitos, no contexto socioeconômico brasileiro, em um rol de variáveis destacadas na literatura sobre fatores de risco e proteção. Para tanto, adotou-se uma metodologia "caso-controle", trabalhando com dados de duas amostras de adolescentes: GI- infratores judicializados; GC- estudantes. Para assegurar à investigação o foco na variável de interesse, ou seja, no "envolvimento sério em prática de delitos", os padrões de conduta delituosa nos grupos foram previamente descritos a partir dos dados levantados por meio da "delinquência autorrevelada" e comparados.
As diferenças médias entre os dois grupos mostram que os adolescentes judicializados (GI) apresentavam, efetivamente, condutas delituosas mais frequentes, mais diversificadas e mais graves/violentas, justificando investigá-los no grupo de interesse.

É importante destacar que os adolescentes estudantes (reunidos no GC) também relataram envolvimento em delitos, ao longo de sua vida (52\% deles relataram pelo menos um delito). Esse dado coaduna com apontamentos da literatura, e remete ao que tem sido denominado "delinquência comum". Em efeito, diversos estudos empíricos evidenciam que grande parte dos adolescentes, especialmente os do sexo masculino, se envolve em delitos, de pouca gravidade, sobretudo na segunda metade da adolescência (Thornberry \& Krohn, 2001; Piquero et al., 2010). Na "delinquência comum", essas condutas, além de apresentarem pouca gravidade e baixa frequência, deixariam de ser manifestas, naturalmente, ao final na adolescência, sendo, dessa forma, consideradas um fenômeno estatisticamente normativo, inerente ao processo desenvolvimental de muitos. A "delinquência comum" não se mostraria associada a fatores de risco, pois seria circunstancial; ao contrário da conduta considerada persistente, atrelada a um “envolvimento sério na prática de delitos". Esse, por seu turno, remete a um padrão de conduta que, além de incluir delitos mais graves, inclui maior diversidade e maior frequência de delitos, se fazendo, via de regra, acompanhar por defasagens psicossociais significativas, atreladas à exposição do adolescente a fatores de risco específicos (mediante ausência ou fragilidade de fatores protetivos) (Piquero et al., 2010). Essa hipótese parece 
confirmada pelos resultados do presente estudo, uma vez que parte das variáveis psicossociais avaliadas, segundo as análises, teria atuado no aumento das chances de o adolescente ser do GI.

Embora o foco do trabalho fosse inicialmente os fatores dinâmicos, percebeu-se que os grupos investigados eram, de partida, significativamente diferentes em algumas variáveis atinentes a fatores estáticos. Uma delas, o Nível Socioeconômico mostrou-se relevante na estimação das chances de os adolescentes pertencerem a um ou a outro grupo. Verificou-se que os níveis mais baixos atuaram aumentando as chances de os adolescentes pertencerem a GI. Baixo status socioeconômico aparece em pesquisas como fator de risco estático em pesquisas, conforme indica a meta-análise realizada por Gendreau, Little e Goggin (1996). É importante sublinhar que baixo status socioeconômico é, em efeito, um fator de risco universal. Para Le Blanc (2003), não é um determinante direto da conduta, mas de condições de vida que podem implicar em mais estresse e em menos proteção ao desenvolvimento, modulando o efeito de outras variáveis diretamente relacionadas à conduta delituosa.

Em seguida, verificou-se que a variável com maior valor OR ponderado foi Ter Filho. Ou seja, o fato de o adolescente mencionar já ser pai. Esse dado não figura nas pesquisas como fator de risco para o sexo masculino. Em algumas investigações, surge como variável significativa para o sexo feminino (Coley, Sims, Dearing, \& Spielvogel, 2017). É fato que a responsabilidade atrelada à parentalidade (maternidade/paternidade), na adolescência, pode funcionar como uma fonte de estresse, considerando as exigências que impõem e as capacidades do(a) adolescente para assumi-las. É fato também que ter filho, no caso dos adolescentes investigados, pode ser pensado como consequência de condutas divergentes, de igual modo ao envolvimento sério em prática de delitos, associadas a um conjunto de fatores de riscos comuns (Rosenbaum, 2013).

Em seguida, a Idade do adolescente mostrou-se relevante, para o compito das chances de pertencer a um ou ao outro grupo. Verificou-se que quanto maior a idade do adolescente, maiores as chance de pertencer a GI. A idade na adolescência, enquanto faixa que vai aproximadamente dos 12 aos 20 anos, não é propriamente um fator de risco, mas sim uma condição de vulnerabilidade para comportamentos de risco, incluindo delitos (Komatsu \& Bazon, 2015). Dados de amostras populacionais mostram que a conduta delituosa obedece a um certo padrão, manifestando-se no início da adolescência, apresentando um pico entre os 15 e 19 anos. Na delinquência comum, conforme o mencionado, ela declina naturalmente, no final da adolescência, tendendo à reabsorção no começo dos 20 anos de idade. $\mathrm{Na}$ delinquência persistente, ela risca perdurar para além do final da adolescência, mediante exposição cumulativa a fatores de risco, alguns, inclusive, novos, mais pertinentes à etapa da vida que se inaugura aí (Piquero et al., 2010). É interessante relembrar que os adolescentes em GI seriam mais velhos que os de GC, com aproximadamente 17 anos, e que estariam em acompanhamento em medidas socioeducativas. $\mathrm{O}$ dado reitera, então, a noção de que, sendo a sua prática de delitos mais frequente, mais diversificada e mais grave, eles teriam um padrão de conduta efetivamente mais persistente. Nesse sentido, a idade, levando em conta o padrão de conduta, denota o histórico do adolescente, em termos de implicação em delitos. Esse, o histórico, sim é um importante fator preditor de conduta futura (Gendreau, Little, \& Goggin, 1996; Cottle, Lee, \& Heilbrun, 2001; Assink et al., 2015).

A última variável alocada no grupo de fatores de risco estático foi Trabalho. Verificou-se que o fato de o adolescente ter declarado já ter trabalhado aumentou a chance de ele pertencer a GI. Aqui, novamente, tem-se a situação de que trabalhar não é uma variável apontada pela literatura como fator de risco para a delinquência. Contudo, a exemplo do que se explanou sobre a parentalidade, é possível que funcione como fonte de estresse para alguns adolescentes, sobretudo se se cogita que os trabalhos que podem ter realizado se dão em condições 
precárias (trabalho braçal/informal), pouco propícias ao pleno desenvolvimento psicossocial do jovem. Ademais, há indicativos que o trabalho, nessas condições, remeta o adolescente precocemente a um "status adulto", propiciando uma maior independência frente às figuras de autoridade familiar (Alves, 2016). Assim, no contexto sociocultural brasileiro, é possível que trabalho possa de fato atuar como fator de risco para a delinquência juvenil. É certo que esse dado confronta o senso comum, a ideia de que trabalhar protege os jovens da implicação em delitos. O dado encontrado, todavia, alinha-se ao de estudos em outras realidades socioculturais, conforme apontamento feito por Uggen e Staff (2001), com base em sua revisão sistemática da literatura. Os autores sublinham que programas de intervenção para adolescentes e jovens adultos infratores, que enfatizam a inserção no mercado de trabalho, não se mostram eficazes, sendo que programas com ênfase no desenvolvimento educacional apresentam melhores resultados.

Focalizando as variáveis alocadas no rol dos fatores considerados risco dinâmico, de natureza pessoal, denota-se, primeiramente, que as variáveis que se destacaram foram Uso Semanal de Maconha e Impulsividade. O abuso de substâncias psicoativas é reiteradamente apontado como um fator de risco para a delinquência juvenil (Andrews \& Bonta, 2006; Assink et al., 2015). No presente estudo, o uso frequente de maconha foi a variável com maior OR de todos, no modelo ajustado, merecendo, portanto, grande destaque. Em outros estudos com amostras brasileiras, existem evidências de que os adolescentes infratores usam mais drogas e têm um padrão de uso mais grave que os adolescentes da população geral (Komatsu, Estevão \& Bazon, 2018). Ainda assim é importante frisar que o estudo apresenta uma limitação nesse ponto, por conta do instrumento utilizado, uma vez que ele avalia apenas o uso frequente da maconha, mas não o de outras drogas, nem mesmo o álcool.

Em relação à Impulsividade, característica relacionada ao baixo autocontrole, essa é considerada um dos mais importantes fatores preditores da delinquência juvenil persistente (Contreras, Molina, \& Cano, 2011). Muitos estudos apontam que dentre os aspectos pessoais que podem ser fatores de risco para a delinquência juvenil, ela é o mais relevante (Wikstrom \& Treiber, 2007). É importante sublinhar que existe uma relação complexa entre impulsividade e uso abusivo de drogas. Existem evidências de que essas variáveis se influenciam mutuamente, ou seja, que o abuso de substâncias favorece escolhas impulsivas e diminuem o controle inibitório, assim como a impulsividade funciona como um fator de risco para o uso abusivo de drogas (Urcelay \& Dalley, 2011).

Quanto às variáveis que remetem a dimensões sociais, destaca-se que pares infratores foi a que apresentou o maior OR. Uma rede de pares composta predominantemente por Pares Antissociais, apresentando problemas de comportamento semelhantes, tem se mostrado como um importante fator de risco para a delinquência juvenil, em inúmeros estudos (Cottle, Lee, \& Heilbrun, 2001; Ortega-Campos, García-García, \& Amenta 2014; Assink et al., 2015). Dentro disso, é preciso considerar a importância que os pares têm nessa etapa do desenvolvimento, enquanto influência socializadora e fonte de apoio social (Bazon \& Estevão, 2012). O pertencimento a um grupo de pares de idade antissociais fomenta a adoção de valores antissociais e de uma atitude favorável à violação das normas, o que certamente ajuda na manutenção da conduta delituosa (Andrews \& Bonta, 2006; Le Blanc, 2006; Bazon \& Estevão, 2012).

Em seguida, a variável com maior OR ajustado foi baixo Desempenho Escolar. Para Le Blanc (2006), ter bom desempenho escolar é um elemento central no sistema de regulação da conduta, no contexto dessa instituição social. Ou seja, o bom desempenho funciona como fator de proteção, propiciando vinculação a essa instituição, facilitando a aceitação de suas normas, por parte dos jovens. Um adolescente que apresenta reiteradamente um baixo desempenho, sente-se menos pertencente ao ambiente escolar. Isso diminui a chance de ele internalizar as suas normas e agir em conformidade a elas (Le Blanc, 2006; Silva, Cianflone \& Bazon, 2016). 
A variável Supervisão Parental, também, inusitadamente, aumentou a chance de o adolescente pertencer a GI no modelo ajustado, indo na direção oposta ao esperado, em se considerando a literatura, que indica que fraca ou inadequada supervisão parental funciona notadamente como fator de risco, ao passo que supervisão existente/adequada pode funcionar como proteção (Hoeve et al., 2009). Tal resultado pode decorrer das características do instrumento. Antes de mais nada, essa escala é a que apresentou menor valor nas avalições de consistência interna, devendo, portanto, os resultados com ela obtidos serem tomados com parcimônia. Ademais, embora as questões que a componham sondem a existência de supervisão, elas não necessariamente avaliam a qualidade da supervisão. Assim, pode-se cogitar que o resultado seja indicativo de uma supervisão inadequada, em razão da qual os adolescentes em GI se sentiriam mais "monitorados", mais "vigiados", sem, contudo, beneficiarem-se dessa prática parental. A supervisão realizada de forma negativa pode ser considerada um fator de risco (Hoeve et al., 2009).

Esse raciocínio se compatibiliza com aquele que decorre dos resultados referentes à variável investimento familiar, a única, segundo o modelo ajustado, atuando na diminuição das chances de o adolescente pertencer à GI, podendo-se pensar em uma atuação dessa como fator protetivo. $\mathrm{O}$ investimento familiar remete ao tempo que a família passa junto, realizando atividades de lazer. O tempo de qualidade passado com membros da família, para além dos efeitos sobre a vinculação familiar, funciona como um modo de estruturar parte do tempo livre do adolescente e, nesse sentido, como uma forma de controle mais efetiva, mais direta, que a própria supervisão (Le Blanc, 2006). De todo modo, é preciso também atentar para o fato de essa escala ter apresentado um baixo valor no índice de confiabilidade.

Quanto às variáveis em relação às quais não se obtiveram evidências de atuação significativa, seja no sentido de aumentar ou de diminuir as chances de os adolescentes pertencerem a GI, no modelo ajustado, pode-se fazer algumas considerações. No plano das variáveis pessoais, tem-se Atitude Violenta, Oposição a Figuras de Autoridade e Valores Antissociais. É interessante notar que os valores do OR bruto, para elas, à exceção de Valores Antissociais, indicaram que aumentariam as chances de o adolescente fazer parte do GI, embora apenas Impulsividade tenha se mantido no modelo ajustado. Supondo a existência de um paralelo entre atitude favorável à violenta, avaliada no estudo, e agressividade, esperava-se que um resultado significativo, uma vez que agressividade é um dos fatores de risco mais importantes para a manutenção da conduta delituosa, inclusive na transição da adolescência para a vida adulta (Assink et al., 2015). Em efeito, agressividade é um risco específico para adolescentes com trajetórias de cometimento de delitos violentos (Reingle, Jennings, \& Maldonado-Molina, 2012; Mulder, Vermunt, Brand, Bullens, \& Marle, 2012; Cuervo, Villanueva, González, Carrión, \& Busquets, 2015). Assim, é possível que essa variável não tenha tido efeito na amostra aqui investigada em função do delineamento do estudo. Não houve a separação dos adolescentes dos subgrupos em função da natureza das condutas delituosas, em meio aos adolescentes apresentando envolvimento sério em delitos.

As variáveis Valores Antissociais e Oposição a Figuras de Autoridade, por seu turno, são aspectos atitudinais relevantes para a compreensão do fenômeno da delinquência juvenil (Hubbard \& Pealer, 2009). Em termos metodológicos também, é preciso considerar que se trata de constructos complexos que, em geral, são avaliados por instrumentos específicos, mais sensíveis, nos estudos da área (Hubbard \& Pealer, 2009; Costa, Komatsu, \& Bazon, 2017). A abordagem mais "direta" deles, no QCJ, pode ter dificultado sua apreensão.

Em relação às variáveis sociais, no plano familiar, aquelas que no modelo ajustado não apresentaram evidências de aumentar ou diminuir as chances de os adolescentes pertencerem a GI, das três avaliadas (Investimento Familiar, Apego Parental e Supervisão 
Parental), apenas o Investimento Familiar se comportou na linha do hipotetizado - ou seja, funcionando como um fator de proteção. Dada a robustez dos estudos que indicam a importância de todas as variáveis da dimensão familiar, é importante cogitar a necessidade de avaliar as práticas parentais de forma mais refinada, também com instrumentos específicos (Andrews \& Bonta, 2006; Hoeve, et al., 2009).

Em relação aos aspectos de rotina, é preciso considerar que Rotina Desestruturada e Pares Antissociais/ Infratores remetem a aspectos da vida de um jovem relacionados entre si, uma vez que adolescentes infratores podem ter uma rotina desestruturada por passarem muito tempo na companhia de pares infratores. Nessa linha, é interessante perceber que a variável Rotina Desestruturada aumentou as chances de o adolescente pertencer a GI, considerando o OR bruto, mas não no modelo ajustado, enquanto Pares Infratores se manteve como uma das variáveis mais significativas na contribuição para as chances de o adolescente pertencer ao grupo de adolescentes infratores, no modelo ajustado.

A variável Religião que, a rigor, poderia funcionar como fator de proteção, não se mostrou significativa. Alguns estudos indicam que a religiosidade pode desempenhar um papel relevante na proteção à delinquência juvenil também por favorecer a vinculação social e a interiorização de um sistema de normas pró-sociais (Le Blanc, 2006; Jang, Bader \& Byron, 2008; Alves, 2016). Ademais, ter uma religião também pode regular a conduta, ao impactar a rotina das pessoas, na medida em que se configura em prática religiosa. Nessa esteira, a variável se comportou de modo equivalente à rotina: se apresentou como um fator de proteção, ao analisar seu OR bruto, mas não quando inserida no modelo ajustado. Por fim, com relação a Apoio Social, variável para o qual se esperava desempenho como fator de proteção, por moderar o impacto de variáveis relacionadas a eventos adversos e por favorecer a vinculação social, não se verificou efeito significativo em nenhuma das análises.
De maneira geral, os resultados mostraram que o envolvimento sério em delitos, na adolescência, no contexto sociocultural brasileiro, também se associa a alguns dos principais fatores colocados pela literatura, reiterando também a concepção que vai na direção de grande parte das pesquisas na área, de que a delinquência juvenil é um fenômeno multideterminado (Andrews \& Bonta, 2006; Le Blanc, 2006). É importante destacar que, à exceção das variáveis "Classe Socioeconômica", "Filhos", "Idade", todas as outras oito variáveis que tiveram resultados significativos no modelo ajustado podem ser consideradas dinâmicas, ou seja, são passíveis de intervenção. Desse modo, políticas com foco na diminuição da delinquência juvenil precisariam abarcar os aspectos que elas representam.

\section{Considerações finais}

Levando em conta que as evidências acerca de fatores de risco específicos associados à delinquência juvenil são bastante sólidas, o presente estudo buscou contribuir nesse sentido, testando algumas variáveis relevantes, em relação ao sexo masculino, no contexto sociocultural brasileiro. Conforme as discussões anteriores, alguns aspectos considerados pela literatura da área apresentaram resultados significativos no presente estudo, embora outros não. Assim, coloca-se a necessidade de aprofundamento nas investigações sobre quais variáveis podem ser consideradas fatores de proteção para a delinquência juvenil no contexto brasileiro, bem como um melhor refinamento naqueles que podem ser considerados fatores de risco. De todo modo, o estudo foi capaz de indicar que o fenômeno da delinquência juvenil é mantido por fatores associados e que a identificação desses fatores, assim como de seus mecanismos, são de grande importância, tanto em termos de desenvolvimento científico, para compreensão do fenômeno, quanto socialmente, para a implementação de políticas públicas/intervenções sensíveis a aspectos que de fato estejam associados à manutenção da conduta delituosa na adolescência. 
Internacionalmente, os estudos já avançaram no sentido de apontar a existência de fatores de risco específicos para trajetórias mais ou menos graves, mesmo tratando-se daqueles adolescentes que apresentam um envolvimento mais sério em prática de delitos (Onifade et al., 2010; Assink el al., 2015). Na revisão de literatura realizada por Galinari, Franco e Bazon (2018), aponta-se a existência consistente de diferentes perfis relacionados à exposição a diferentes conjuntos de fatores de risco e de proteção. Isso pode ser considerado uma limitação do presente estudo, sendo importante que em pesquisas futuras se busque por mais refinamento na composição das amostras estudadas. Estudos internacionais também já indicam subconjuntos específicos de fatores relacionados ao início de uma trajetória da conduta delituosa persistente, outros fatores que são específicos à sua manutenção desta e também à desistência da trajetória (Moffit, 2018). Esse avanço remete, obviamente, a um outro limite da presente pesquisa. Esse refinamento nos estudos longitudinais, com grandes amostras, representam uma grande contribuição para a implementação de políticas públicas eficazes, uma vez que os fatores que precisam ser focalizados para prevenção primária podem não ser os mesmos para prevenção secundária.

\section{Referências}

ABEP. Critério Padrão de Classificação Econômica Brasil, 2017.

Andrews, D. A. \& Bonta, J. (2006) The Psychology of Criminal Conduct. LexisNexis: Newark.

Assink, M., van der Put, C., Hoeve, M., de Vries, S., Stams, G. \& Oort, F. (2015). Risk factors for persistent delinquent behavior among juveniles: A meta-analytic review. Clin Psychol Rev., 42, 47-61. https://doi. org/10.1016/j.cpr.2015.08.002

Bazon, M. R., \& Estevão, R. (2012). Juvenile Criminal Behavior and Peers' Influences: A Comparative Study in the Brazilian Context. Universitas Psychologica, 11, 1157- 1166. https://doi.org/10.1016/j.cpr.2015.08.002
Brasil, Ministério dos Direitos Humanos (MDH). (2018). Levantamento anual SINASE 2016. Brasília: Ministério dos Direitos Humanos. https://doi.org/10.11606/t.2.2010. tde-26012011-151013

Coley, R. L., Sims J., Dearing, E \& Spielvogel, B. (2017). Locating economic risks for adolescent mental and behavioral health: poverty and affluence in families, neighborhoods, and schools. Child Dev. https://doi. org/10.1111/cdev.12771

Contreras, L., Molina, V., \& Cano, M. C. (2011). In search of psychosocial variables linked to the recidivism in young offenders. The European Journal of Psychology Applied to the Legal Context, 3, 77-88.

Cottle, C. C., Lee, R. J., \& Heilbrun, K. (2001). The Prediction of Criminal Recidivism in Juveniles: A Meta-Analysis. Criminal Justice and Behavior, 28(3), 367-394. https://doi.org/10.1177/0093854801028003005

Costa, R., Komatsu, A. V., \& Bazon, M. R. (2017). Psychological Assessment of Adolescent Offenders. International Annals of Criminology, 0: 1-20

Cuervo, K., Villanueva, L., González, F., Carrión, C., $\&$ Busquets, P. (2015). Characteristics of young offenders depending on the type of crime. Psychosocial Intervention, 24(1), 9-15. https://doi.org/10.1016/j. psi.2014.11.003.

Dickens, G. L. \& O'Shea, L. E. (2017). Protective Factos in Risk Assessment Schemes for Adolescents in Mental Health and Criminal Justice Populations: A Systematic Review and Meta-Analysis of their Predictive Efficacy. Adolescent Research Review, 3(1), 95-112. https://doi. org/10.1007/s40894-017-0062-3

Eisenstein, E., \& Souza, R. P. de (1993) Situações de risco à saúde de crianças e adolescentes. Petrópolis: Vozes.

French, Jan Hoffman. "Repensando a Violência Policial no Brasil: Desmascarando o Segredo Público da Raça*."Revista TOMO31(2017): 9-40. https://doi. org/10.21669/tomo.v0i0.7648

Galinari, L. S., Franco, M. G. O., Bazon, M. R. (2018). Perfis de Risco em Delinquência Juvenil: implicações para avaliação e intervenção. In Cândido da Agra, Marcus Alan Gomes. (Orgs.). Criminologia Integrativa: contributos para uma comunidade criminológica de língua portuguesa. (1ed., pp. 251-272). Belo Horizonte: DPlácido. 
Gendreau, P., Little, T. \& Goggin, C. (1996). A meta-analysis of the predictors of adult offender recidivism: What works!. Criminology, 34(4), 575-608. https://doi. org/10.1111/j.1745-9125.1996.tb01220.x

Hoeve, M., Dubas, J., Eichelsheim, V. van der Laan, P. Smeenk, W. \& Gerris, J. (2009). The relationship between parenting and delinquency: a meta-analysis. J Abnorm Child Psychol, 37(6), 749-759. https://doi. org/10.1007/s10802-009-9310-8

Hosmer, D.W. \& Lemeshow, S. (2000) Applied logistic regression. (2nd ed.). New York: John Wiley \& Sons. https://doi.org/10.1002/0471722146

Hubbard, D. J., \& Pealer, J. (2009). The Importance of Responsivity Factors in Predicting Reductions in Antisocial Attitudes and Cognitive Distortions Among Adult Male Offenders. The Prison Journal, 89(1), 79-98. https://doi.org/10.1177/0032885508329987.

Jang, S. J., Bader, C., Byron, J. (2008). The Cumulative Advantage of Religiosity in Preventing Drug Use. Journal of Drug Issues, 38. https://doi. org/10.1177/002204260803800306

Komatsu, A.V. \& Bazon, M.R. (2015). Caracterização de adolescentes do sexo masculino em relação a comportamentos antissociais. Revista Latinoamericana de Ciencias Sociales, Niñez y Juventud, 13(2): 725-735. https://doi.org/10.11600/1692715x.13212210814

Komatsu, A. V. (2014). Comportamentos antissociais em adolescentes do sexo masculino: um estudo exploratório na cidade de Ribeirão Preto - SP (Dissertação de Mestrado). Universidade de São Paulo, Ribeirão Preto, SP.

Komatsu, A. V., \& Bazon, M. R. (2017b). Personal differences among Brazilian adolescents with different levels of criminal engagement. International Journal of Criminology \& Sociology, 6: 65-74. https:// doi.org/10.6000/1929-4409.2017.06.07

Le Blanc, M. \& Fréchette, M. (1989). Male Criminal Activity from Childhood through Youth: Multilevel and developmental perspectives. In Farrington, D. \& Blumstein, A. (Eds.). Research in Criminology. New York: Springer-Verlag. https://doi.org/10.1007/978-1$\underline{4612-3570-5 \_1}$
Le Blanc, M. (2003). La conduite délinquante des adolescents: son développement et son explication. In M. Leblanc, M. Ouimet, \& D. Szabo. Traité de criminologie empirique. Montréal: Les Presses de l'Université de Montréal. https://doi.org/10.4000/books.pum.6658

Le Blanc, M. (2006). Self-control and social control of deviant behavior in context: development and interactions along the life course. In P. O. H. Wikström \& R. J. Sampson (Eds.). The explanation of crime: context, mechanisms, and development. (pp. 195-242). Cambridge, UK: Cambridge University Press. https:// doi.org/10.1017/cbo9780511489341.007

Maruschi, M. C., Estevão, R. \& Bazon, M. R. (2013). Aplicação de medidas socioeducativas em adolescentes: Avaliação auxiliar às tomadas de decisão. Psicologia PUCRS, 44(3), 453-463.

Moffitt, T. E. (2018) Male antisocial behaviour in adolescence and beyond. Nature Human Behaviour, 2(3), 177-186. https://doi.org/10.1038/s41562-018-0309-4

Mulder, E., Vermunt, J., Brand, E., Bullens, R., \& Marle, H. (2012). Recidivism in subgroups of serious juvenile offenders: Different profiles, different risks? Criminal Behaviour and Mental Health. https://doi. org/10.1002/cbm.1819

Mullis, R. L., Cornille, T. A., Mullis, A. K., \& Huber, J. (2004). Female Juvenile Offending: A Review of Characteristics and Contexts. Journal of Child and Family Studies, 13(2), 205-218. http://dx.doi.org/10.1023/B:JCFS.0000015708.71295.2a

Onifade, E., Smith Nyandoro, A., Davidson II, W. S., \& Campbell, C. (2010). Truancy and patterns of criminogenic risk in a young offender population. Youth Violence and Juvenile Justice, 8(1), 3-18. https://doi. org/10.1177/1541204009338251

Ortega-Campos, García-García \& Armenta (2014). Meta-Analysis of juvenile criminal recidivism: Study of Spanish research. Revista Mexicana de Psicologia. 31(2), 111-123.

Piquero, A. R., Farrington, D. P., Nagin, D. S. \& Moffitt, T. (2010). Trajectories of offending and their relation to life failure in late middle age: Findings from the Cambridge study in delinquent. Journal of research in crime and delinquency, 47(2), 151-173. https://doi. org/10.1177/0022427809357713 
Reingle, J. M., Jennings, W. G., \& Maldonado-Molina, M. M. (2012). Risk and protective factors for trajectories of violent delinquency among a nationally representative sample of early adolescents. Youth Violence and Juvenile Justice. https://doi.org/10.1177/1541204011431589

Rosenbaum, J. (2013). An examination of psychopathy, promischuity and other risky sexual behavior over time (tese de doutorado). University of Alabama, Alabama. Silva, J. L., Cianflone, A. R. L., \& Bazon, M. R. (2016) School bonding of adolescent offenders. Paidéia (Ribeirão Preto), 26, 91-100. https://doi.org/10.1590/1982$\underline{43272663201611}$

Thornberry, T. P. \& Krohn, M. D. (2001). Development of Delinquency: An Internactional Perspective. Handbook of youth and justice, 289-305. https://oi. org/10.1007/978-1-4615-1289-9 15.

Uggen, C., \& Staff, J. (2001). Work as a turning point for criminal offenders. Corrections management quarterly, 5(4), 1-16.

Urcelay G. P., Dalley J.W. (2011) Linking ADHD, Impulsivity, and Drug Abuse: A Neuropsychological Perspective. In Stanford C., Tannock R. (Eds). Behavioral Neuroscience of Attention Deficit Hyperactivity Disorder and Its Treatment. Current Topics in Behavioral Neurosciences (9). Springer, Berlin: Heidelberg. https:// doi.org/10.1007/7854 2011 119

Wikstrom, P. H., Treiber, K. (2007) The Role of Self-Control in Crime Causation. European Journal of Criminology, 4(2), 237-264. https://doi. org/10.1177/1477370807074858

Recebido em: 6/5/2019.

Aprovado em: 14/8/2019.

Publicado em: $\mathrm{xx} / \mathrm{x} / \mathrm{xxxx}$

Endereço para correspondência: Profa Dra Marina Rezende Bazon (Universidade de São Paulo Av.

Bandeirantes, 3900, CEP 14040-901, Ribeirão Preto - SP - Brasil; (16) 3315-3830; mbazon@ffclrp.usp.br

\section{Nome: Lais Sette Galinari}

E-mail:laissette@gmail.com
Titulação Acadêmica: Mestranda no Programa de Pós-Graduação em Psicologia da Faculdade de Filosofia, Ciências e Letras de Ribeirão Preto Afiliação Institucional: Universidade de São Paulo (USP)

\section{Nome: Íris Daniela Arruda Vicari}

E-mail:irispsicorp@gmail.com

Titulação Acadêmica: Mestranda no Programa de Pós-Graduação em Psicologia da Faculdade de Filosofia, Ciências e Letras de Ribeirão Preto Afiliação Institucional: Universidade de São Paulo (USP)

\section{Nome: Prof ${ }^{\mathrm{a}}$. Dr ${ }^{\mathrm{a}}$. Marina Rezende Bazon}

E-mail:mbazon@ffclrp.usp.br

Titulação Acadêmica: Livre docente e Professora

Associada do Departamento de Psicologia da FFCLRP-USP

Afiliação Institucional: Universidade de São Paulo (USP) 